\title{
High-resolution spectroscopy for Cepheids distance determination ${ }^{\star}$
}

\section{Time series of $\mathrm{H} \alpha$ line profiles}

\author{
N. Nardetto ${ }^{1}$, J. H. Groh ${ }^{1}$, S. Kraus ${ }^{1}$, F. Millour ${ }^{1}$, and D. Gillet ${ }^{2}$ \\ ${ }^{1}$ Max-Planck-Institut für Radioastronomie, Auf dem Hügel 69, 53121 Bonn, Germany \\ e-mail: nardetto@mpifr-bonn.mpg.de \\ 2 Observatoire de Haute-Provence, 04870 Saint-Michel l'Observatoire, France
}

Received 15 February 2008 / Accepted 1 April 2008

\begin{abstract}
Context. In recent years, infrared interferometry has revealed the presence of faint dusty circumstellar envelopes (CSE) around Cepheids. However the size, shape, chemical nature, and the interaction of the CSE with the star itself are still under investigation. The presence of a CSE might have an effect on the angular diameter estimates used in the interferometric Baade-Wesselink and surface-brightness methods of determining the distance of Cepheids.

Aims. By studying $\mathrm{H} \alpha$ profiles as a function of the period, we investigate the permanent mass loss and the CSE around Cepheids. Our high spectral- and time-resolution data, combined with a very good $\mathrm{S} / \mathrm{N}$, will be useful in constraining future hydrodynamical models of Cepheids atmosphere and their close environment.

Methods. We present HARPS ${ }^{\star \star}$ high-resolution spectroscopy $(R=120000)$ of eight galactic Cepheids: $\mathrm{R}$ Tra, S Cru, Y Sgr, $\beta$ Dor, $\zeta$ Gem, RZ Vel, $\ell$ Car, and RS Pup, providing a good period sampling $(P=3.39 \mathrm{~d}$ to $P=41.52 \mathrm{~d})$. The $\mathrm{H} \alpha$ line profiles are described for all stars using a 2D (wavelength versus pulsation phase) representation. For each star, an average spectral line profile is derived, together with its first moment ( $\gamma$-velocity) and its asymmetry ( $\gamma$-asymmetry).

Results. Short-period Cepheids show $\mathrm{H} \alpha$ line profiles following the pulsating envelope of the star, while long-period Cepheids show very complex line profiles and, in particular, large asymmetries. We find a new relationship between the period of Cepheids and their $\gamma$-velocities and -asymmetries. These results may be related to the dynamical structure of the atmosphere and to a permanent mass loss of Cepheids. In particular, we confirm for $\ell$ Car a dominant absorption component whose velocity is constant and nearly of zero $\mathrm{km} \mathrm{s}^{-1}$ in the stellar rest frame. This component is attributed to the presence of circumstellar envelope.

Conclusions. To understand these very subtle $\gamma$ effects, fully consistent hydrodynamical models are required, including pulsating and evolutionary theories, convective energy transport, adaptive numerical meshes, and a refined calculation of the radiative transfer.
\end{abstract}

Key words. techniques: spectroscopic - stars: atmospheres - stars: oscillations - stars: variables: Cepheids - stars: distances

\section{Introduction}

Cepheids are key astrophysical objects due to their well-known period-luminosity $(P L)$ relationship. Based on this relationship, a multi-decade work has reached cosmologically significant extragalactic distances (see Hubble Space Telescope Key Project, Freedman et al. 2001). In particular, two methods have recently been used to calibrate the $P L$ relationship, namely the infrared surface brightness (IRSB) and the interferometric Baade-Wesselink (IBW) methods of determining the distance of Cepheids (Fouqué et al. 2007).

The basic principle behind these methods is to compare the linear and angular size variation of a pulsating star to derive its distance through a simple division. The caveat is that interferometric (IBW) or photometric (IRSB) measurements in the

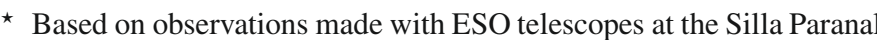
Observatory under program IDs 072.D-0419 and 073.D-0136.

$\star \star$ High Accuracy Radial velocity Planetary Search project developed by the European Southern Observatory.
}

continuum lead to angular diameters corresponding to the photospheric layer, while the linear stellar radius variation is deduced from spectroscopy, i.e., based on line-forming regions that form in higher layers of the atmosphere. Thus, radial velocities $V_{\mathrm{rad}}$, which are derived from line profiles, include the integration in two directions: over the stellar surface through limb darkening, and over the atmospheric layers through velocity gradients. All these phenomena are currently merged into one specific quantity, generally considered as constant with time: the projection factor $p$, defined as $V_{\text {puls }}=p V_{\text {rad }}$, where $V_{\text {puls }}$ is defined as the $p h o$ tospheric pulsation velocity (Nardetto et al. 2004). Then, $V_{\text {puls }}$ is integrated with time to derive the photospheric radius variation. The precision in the distance currently obtained with the IBW and IRSB methods is a few percent; however, they remain strongly dependent on the projection factor. If a constant projection factor is used (generally $p=1.36$ for all stars, Burki et al. 1982) to derive the $P L$ relationship, errors of 0.10 and 0.03 on the slope and zero-point of the $P L$ relationship can be introduced. It means that distances can be overestimated by $10 \%$ for long-period Cepheids (Nardetto et al. 2007a, hereafter Paper II). 

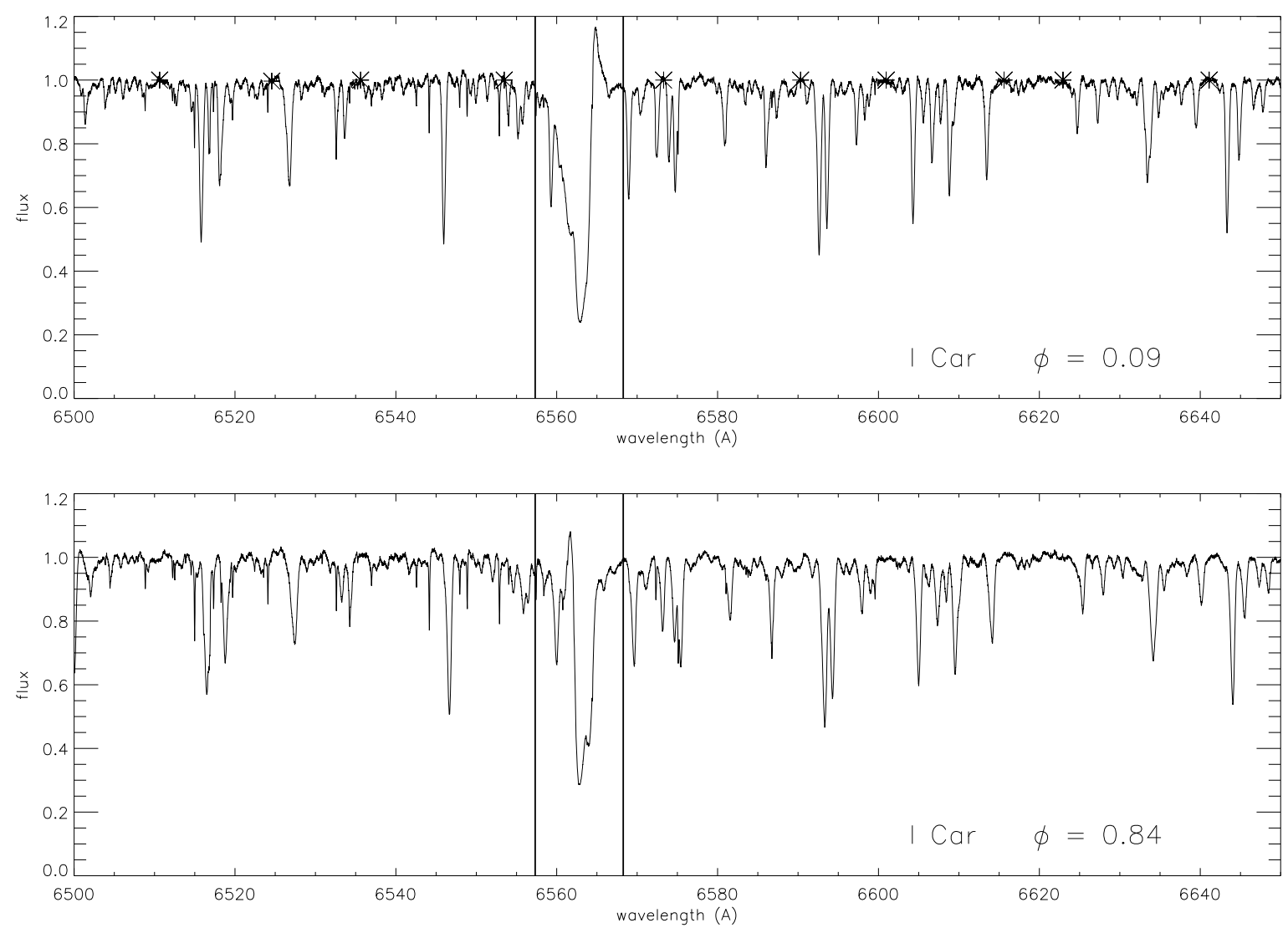

Fig. 1. Typical HARPS spectrum ( $\ell$ Car, $\phi=0.09$ and $\phi=0.84$ ) around $\mathrm{H} \alpha$. In this study, we focus on the $[-250,250] \mathrm{km} \mathrm{s}^{-1}$ velocity range represented in the figure by the two vertical lines, while the whole wavelength range $6500-6650 \AA$ was used in the normalization process. Ten points (crosses on the upper panel) are used in the interpolation of the continuum. Within the $[-250,250] \mathrm{km} \mathrm{s}^{-1}$ velocity range, we clearly see that the blue wing of the $\mathrm{H} \alpha$ line is blended by a metallic line identified as either Fe I $6559.763 \AA$ or possibly Ti II $6559.588 \AA$.

In recent years, infrared interferometry has revealed the presence of faint (few percent of near-infrared continuum flux), dusty circumstellar envelopes (CSE) around Cepheids (Kervella et al. 2006; Mérand et al. 2006, 2007). However, the size (the CSE is 3-4 times larger than the star), shape, chemical nature, and the interaction of the CSE with the star itself are still under investigation. Such envelopes have a non-negligible effect on the angular diameter estimates used in the IBW and IRSB methods of determining the distance of Cepheids. If the CSE is not considered, the distance can be underestimated by a few percent. In addition, Mérand et al. (2007) show a relationship between the period of the Cepheid and the brightness of the CSE compared to the stellar near-infrared continuum flux. From these results, one cannot exclude a link between the period, the mass loss, and the presence of a circumstellar shell around Cepheids. The knowledge of these CSE is particularly important in the case of the bright, long period cepheids, which can be used to improve the primary distance scale to Virgo and beyond. Recently, Kervella et al. (2008) determine the distance of the long period Cepheid RS Pup from its light echoes, while Nardetto et al. (2006, hereafter Paper I) found a broadening of the metallic spectral lines at certain phases that could indicate the presence of a strong velocity gradient (compression or shock wave). In this context, studying and understanding the $\mathrm{H} \alpha$ profile variations of Cepheids as a function of the period is a key. This spectral line is indeed sensitive both to pulsation and the putative hydrogen circumstellar medium.
We present the time series of $\mathrm{H} \alpha$ profiles for eight Cepheids, with a very high spectral resolution and a good time coverage. Our aim is not to provide a full picture of the mechanisms involved in the formation of $\mathrm{H} \alpha$ line profiles, but mainly to characterize them and to investigate a relationship with the period of the star. With such high $\mathrm{S} / \mathrm{N}$, high-resolution data will be useful for supporting future hydrodynamical models of Cepheids' atmosphere and their close environment. In Sect. 2, we present our data and the $\mathrm{H} \alpha$ profile of the studied Cepheids. Section 3 presents an analysis in terms of $\gamma$-velocities and -asymmetries and Sect. 4 is devoted to the particular case of $\ell$ Car.

\section{The high-resolution $\mathrm{H} \alpha$ line profiles of Cepheids}

Ten Cepheids were observed with the HARPS spectrometer: R Tra, S Cru, Y Sgr, X Sgr, $\beta$ Dor, $\zeta$ Gem, Y Oph, RZ Vel, $\ell$ Car and RS Pup, having a good period sampling from $P=3.39 \mathrm{~d}$ to $P=41.52 \mathrm{~d}$ (Paper I). X Sgr is an atypical Cepheid presenting several components in the spectral line profiles, and has been studied separately by Mathias et al. (2006). Y Oph is not studied in detail here due to its insufficient phase coverage. Thus, we consider 8 Cepheids in this paper. The spectral resolution is $R=\frac{\lambda}{\Delta \lambda}=120000$ and the average $\mathrm{S} / \mathrm{N}$ is 300 per pixel. We used the standard ESO/HARPS pipeline reduction package for bias subtraction, flat-fielding, and order extraction. 
We paid special attention to the normalization process. For each spectrum, the continuum was defined by interpolating ten points (using a spline function), chosen carefully over the 6500-6650 Å wavelength range (Fig. 1). We consequently estimate the uncertainty on the continuum level to be about $1 \%$ (i.e. around 3 times the average noise).

Our observations generally spread over a few pulsation cycles (2 to 4). Assuming negligible cycle-to-cycle variations, our total dataset provides a very good pulsation phase coverage. Journal of observations and online data are available in Nardetto et al. (2007b). Combining very high spectral resolution and good time coverage, we derive 2D maps using an interpolation method based on the so-called Delaunay triangles (see "triangulate" function in IDL $^{1}$ and Renka 1982). The wavelength and pulsation phase sampling of the maps are respectively $0.06 \AA$ and 0.02. To describe $\mathrm{H} \alpha$ line profiles $(6562.797 \AA)$, we classified the Cepheids in our sample in three groups: short- (R TrA, $\mathrm{S}$ Cru, and Y Sgr), medium- ( $\beta$ Dor and $\zeta$ Gem), and longperiod Cepheids (RZ Vel, $\ell$ Car and RS Pup), corresponding to Figs. $2-4$, respectively. All diagrams are given in the stellar rest frame with positive velocities corresponding to receding motion. The data were duplicated over 2 cycles for clarity. The $\gamma$-velocities (average value of the radial velocity curve derived from a metallic line) were corrected using the Galactic Cepheid Database of the David Dunlap Observatory ${ }^{2}$ (Fernie et al. 1995). The plots are presented over the $[-250,250] \mathrm{km} \mathrm{s}^{-1}$ velocity range around $\mathrm{H} \alpha$, to avoid complex line blendings on the extreme parts of the line wings (Fig. 1). The only strong remaining metallic line within this velocity range is Fe I $6559.763 \AA$ (or possibly Ti II $6559.588 \AA$ ). The vertical lines that sometimes appear faint (for instance at $-220 \mathrm{~km} \mathrm{~s}^{-1}$ for $\beta$ Dor or $+170 \mathrm{~km} \mathrm{~s}^{-1}$ for $\ell$ Car) are telluric lines, and they have negligible influence on our results.

Following our three groups, several features should be mentioned:

- Short-period Cepheids [3 to 6 days] confirm the observations by Wallerstein (1972) that in stars with periods less than $13 \mathrm{~d}$, the $\mathrm{H} \alpha$ line profiles behaves similar to other absorption lines. In the left part of each panel, one can indeed use the track corresponding to the Fe I $6559.763 \AA$ metallic line profile (around $-160 \mathrm{~km} \mathrm{~s}^{-1}$ ) as a reference. This track is quite faint for short-period stars, but clearly visible for medium- and long-period Cepheids. Otherwise, these shortperiod Cepheids show very similar profiles from one star to the next.

- Medium-period Cepheids [around 10 days], namely $\beta$ Dor and $\zeta$ Gem, are different from the short-period Cepheids simply by the shape of their radial velocity curve. These stars are indeed very close to the $\frac{P_{2}}{P_{0}}=0.5$ resonance, where $P_{0}$ and $P_{2}$ are respectively the periods corresponding to the fundamental and the second overtone modes (Kovacs et al. 1990). We even notice that $\zeta$ Gem $(P=9.8 \mathrm{~d})$ is less affected by the resonance compared to $\beta$ Dor $(P=10.1 \mathrm{~d})$.

- Long-period Cepheids [20 to 42 days] show a clear asymmetry in the $\mathrm{H} \alpha$ line profiles, which is present during most of the pulsation cycle. The line asymmetry is studied in detail for all stars of our sample in the next section. Long-period Cepheids also present a complicated structure consisting of multi-components in absorption or in emission, which might be explained by means of the presence of a turbulent layer

\footnotetext{
1 Interface Definition Language.

2 http://www.astro.utoronto.ca/DDO/research/cepheids/
}
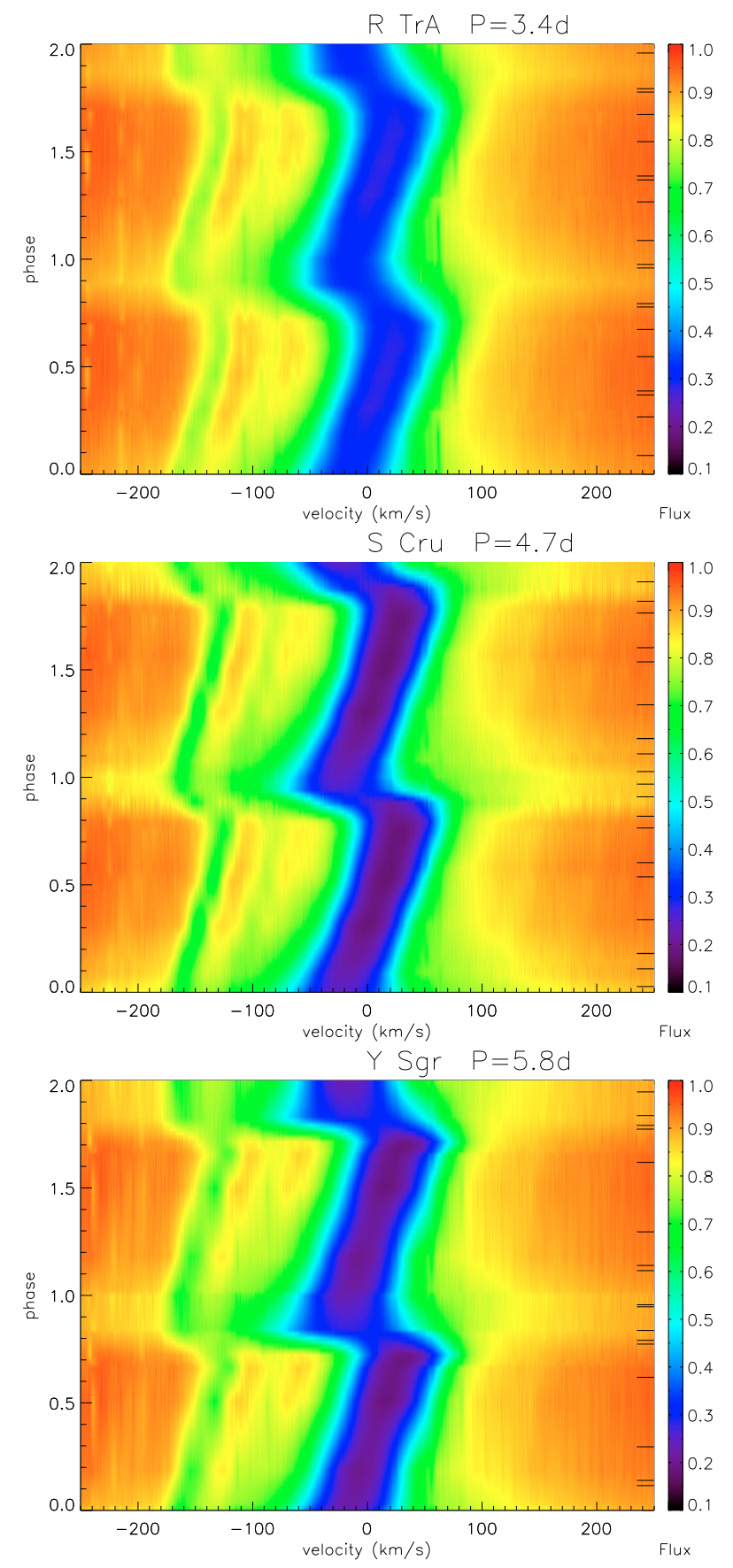

Fig. 2. $\mathrm{H} \alpha$ line profiles of short-period Cepheids $\mathrm{R} \operatorname{TrA}, \mathrm{S}$ Cru and Y Sgr, ordered by increasing period from top to bottom. Time series of HARPS spectra are interpolated to provide a 2-dimensional map of the $\mathrm{H} \alpha$ profile in the $[-250,250] \mathrm{km} \mathrm{s}^{-1}$ velocity range. Diagrams are given in the stellar rest frame with positive velocities corresponding to receding motion (red-shifted). The pulsation phase is indicated on the left edge of each panel and on the right we quoted the pulsation phases corresponding to our observations (data are duplicated over two pulsation cycles for clarity). For each star the color bar (indicating the flux) is from 0.1 to 1.0 .

(maybe due to a shockwave) in the upper atmosphere of the star, and by the presence of a circumstellar shell (see e.g. Rodgers \& Bell 1968; Grenfell \& Wallerstein 1969; Schmidt 1970; Wallerstein 1972, 1979, 1983, 1992; Böhm-Vitense \& Love 1994). In addition, as shown by detailed models of the fine structure of radiative shocks (Fadeyev \& Gillet 2004), 

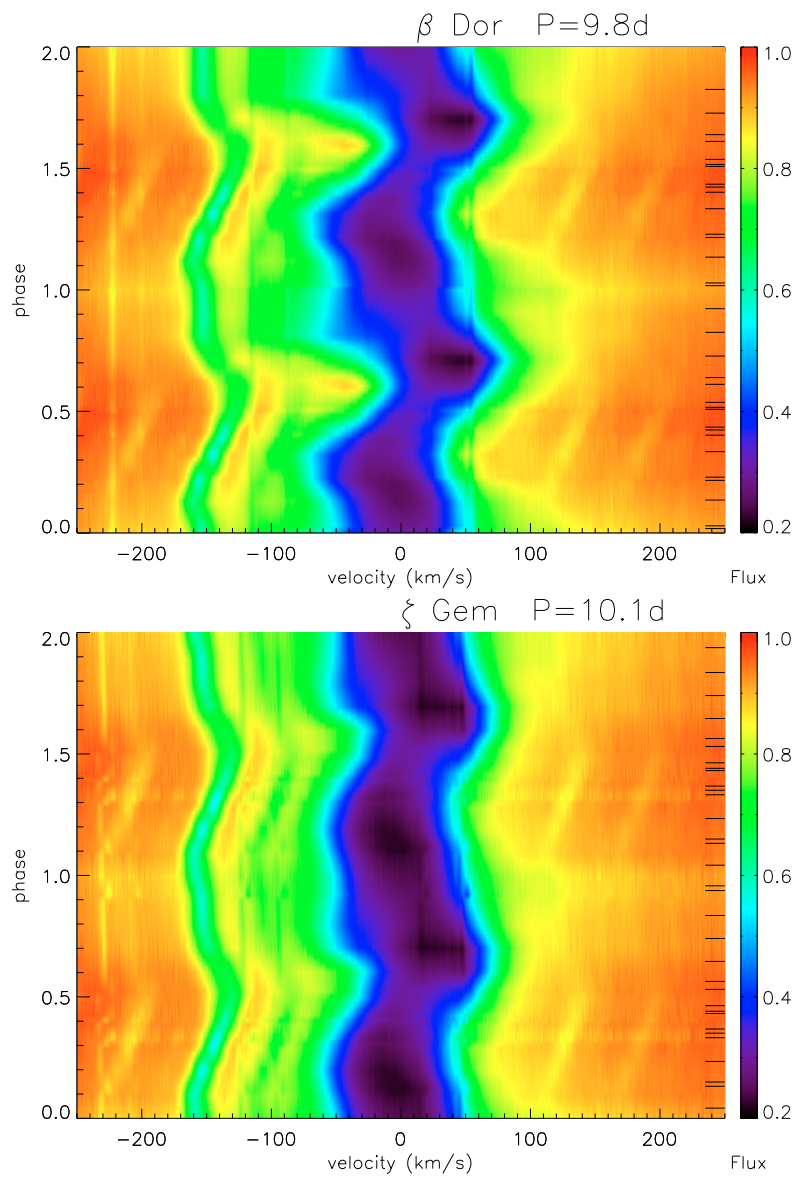

Fig. 3. Same as Fig. 2 but for the medium-period Cepheids $\beta$ Dor and $\zeta$ Gem. For each star the color bar is from 0.2 to 1.0 .

hydrogen emission lines might also be formed within the deexcitation region of the shockwave. We discuss the particular case of $\ell$ Car in Sect. 4.

\section{A relationship between the period and the $\gamma$-velocities and -asymmetries}

The $\gamma$-velocity (resp. $\gamma$-asymmetry) is usually defined as the average of the radial velocity curve (resp. line asymmetry curve) over a complete pulsation cycle. An interpolation over the pulsation phase is then absolutely required to derive the $\gamma$-velocity (resp. $\gamma$-asymmetry) properly. This method is referred hereafter as the 1D method. Physically, the $\gamma$-velocity is either related to star motion itself, consequently to the kinematical structure of our Galaxy, or to the dynamical structure of the spectral lineforming region. For instance, if the $\gamma$-asymmetry is not zero, then the corresponding $\gamma$-velocity is at least partially due to an intrinsic physical property of the Cepheid. Such a study has already been done by Nardetto et al. (2008, hereafter Paper III) for metallic lines.

We apply a new method here for deriving the $\gamma$-velocities $\left(V_{\gamma}\right)$ and asymmetries $\left(A_{\gamma}\right)$. For each star, we first derive an unweighted average spectral line profile over all pulsation phases using the interpolated profiles of the $2 \mathrm{D}$ map. Once the average spectral line profiles are derived for all stars (Fig. 5), the metallic line profile between -180 and $-110 \mathrm{~km} \mathrm{~s}^{-1}$ is removed using a
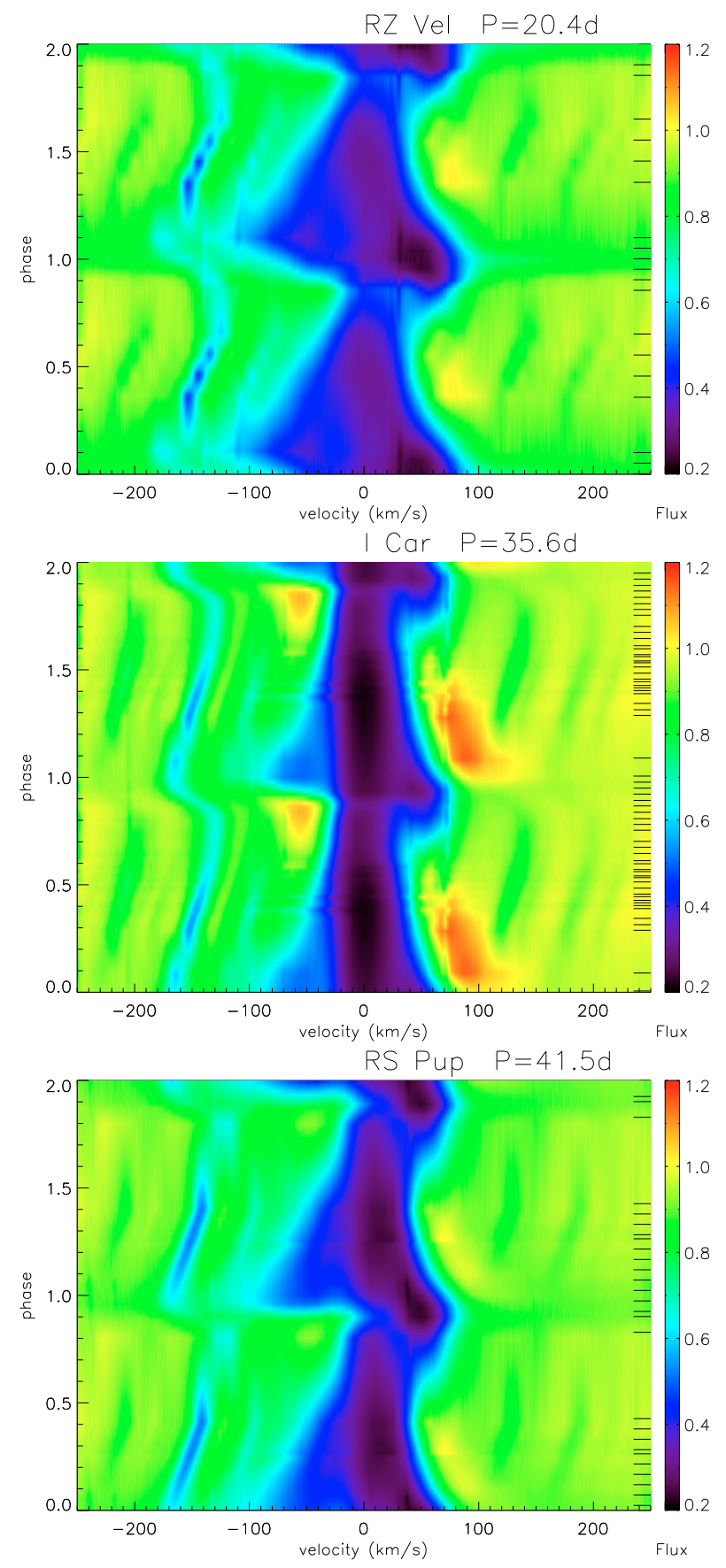

Fig. 4. Same as Fig. 2 but for the long-period Cepheids RZ Vel, $\ell$ Car and RS Pup. For each star the color bar is from 0.2 to 1.2.

linear interpolation (in the wavelength space). Then, we use the first-moment definition to derive $V_{\gamma}$ :

$V_{\gamma}=\frac{\int_{\text {line }} \lambda S(\lambda) \mathrm{d} \lambda}{\int_{\text {line }} S(\lambda) \mathrm{d} \lambda}$,

where $S(\lambda)$ is the observed average line profile over the $[-250,250] \mathrm{km} \mathrm{s}^{-1}$ velocity interval. We insist on this firstmoment definition, since it is absolutely required to allow important comparisons between the $\gamma$-velocities of different Cepheids. Indeed, it is the only method (compared to the Gaussian fit or pixel minimum methods) that provides a radial velocity independent of the rotation (projected on the line of sight) and the natural width of the spectral line (Burki et al. 1982; Paper I). To 
measure the $\mathrm{H} \alpha$ line asymmetry, we use here the method first mentioned by Sabbey et al. (1995), which uses the entire line profile. An asymmetry percentage was determined from the difference and sum of the areas of the red and blue profile halves, $\frac{\left(A_{\text {red }}-A_{\text {blue }}\right)}{A_{\text {total }}} * 100$, and the spectral channel with minimum flux was used to define the line center. The method used in Paper I, based on two semi-Gaussians, is not applicable here, providing reduced $\chi^{2}$ higher than 200 .

Even if this 2D-method is not strictly equivalent to the previous 1D-method, this approach is more robust and also easier to apply in the context of the $\mathrm{H} \alpha$ profiles. A comparison between both methods was possible for the short- and medium-period Cepheids, and we found good consistencies at a level of $2 \mathrm{~km} \mathrm{~s}^{-1}$ for the $\gamma$-velocity and $2 \%$ for the line asymmetry. However, the 1D-method cannot be applied for long-period Cepheids. Indeed, in this case, the $\mathrm{H} \alpha$ profiles are complex, with multiple components in absorption and/or in emission (Fig. 1), and with a significant variation from one pulsation phase to the other, implying different problems: (1) there is no obvious definition of the line asymmetry, and (2) the resulting radial velocity and asymmetry curves are extremely difficult to interpolate, making $V_{\gamma}$ and $A_{\gamma}$ uncertain. By deriving our $\mathrm{H} \alpha 2 \mathrm{D}$ maps, we make a very efficient $2 \mathrm{D}$ interpolation based on triangles, which is more robust than the usual 1D interpolation over the pulsation phase.

Statistical uncertainties (given the high $\mathrm{S} / \mathrm{N}$ and the high spectral resolution) are less than $1 \mathrm{~km} \mathrm{~s}^{-1}$ and $1 \%$, respectively. Uncertainties on $V_{\gamma}$ and $A_{\gamma}$ are clearly dominated by systematic errors. First, the choice of the velocity window $[-250,250] \mathrm{km} \mathrm{s}^{-1}$ can modify the $\gamma$-velocity and $\gamma$-asymmetry by $5 \mathrm{~km} \mathrm{~s}^{-1}$ and $5 \%$, respectively, while the choice of the continuum (within 3\%) also has an impact of about $6 \mathrm{~km} \mathrm{~s}^{-1}$ and $6 \%$, respectively. These systematic errors mainly come from the limits of the spectral line being not clearly determined because of many blends that cannot be corrected satisfactorily (see Fig. 1). In the case of RS Pup, the phase coverage also shows a gap between 0.4 and 0.8 , which keeps the 2D-interpolation from being totally secure: a break is observed in the track of the Fe I $6559.763 \AA$ metallic line near $\phi=0.55$. However, in the case of the $\mathrm{H} \alpha$ line, the general tendency remains satisfactory (only a small unavoidable artefact is observed on the red wing at $\phi=0.6$ ), which makes the average spectral line quite relevant. We quantify the impact of the phase coverage on $V_{\gamma}$ and $A_{\gamma}$ by artificially removing the observed spectral lines profiles of $\ell$ Car found between phases 0.4 and 0.8 . We find a decrease in the absolute values of $V_{\gamma}$ and $A_{\gamma}$ of about $2 \mathrm{~km} \mathrm{~s}^{-1}$ and $2 \%$, respectively. For other stars than RS Pup, the phase coverage seems satisfactory. Global uncertainties on $V_{\gamma}$ and $A_{\gamma}$ are finally estimated to be $8 \mathrm{~km} \mathrm{~s}^{-1}$ and $8 \%$ for all stars.

Following this strategy, our observations suggest a linear relationship between the period of the star and the $\mathrm{H} \alpha \gamma$-velocities and $\gamma$-asymmetries:

$V_{\gamma}\left[\mathrm{km} \mathrm{s}^{-1}\right]=[-9.1 \pm 3.3] \log P+[12.1 \pm 8.4]$,

$A_{\gamma}[\%]=[5.1 \pm 3.5] \log P-[0.6 \pm 8.4]$.

These relationships are presented in Fig. 5. The reduced $\chi^{2}$ are 1.8 and 0.7. First, the anti-correlation between these two relationships follows the expectation that the more the line is asymmetric, the larger the first moment of the spectral line. Therefore, it clearly shows that the $\gamma$-velocities obtained are due to an intrinsic physical property in Cepheids and not to their residual individual motions within the Galaxy.
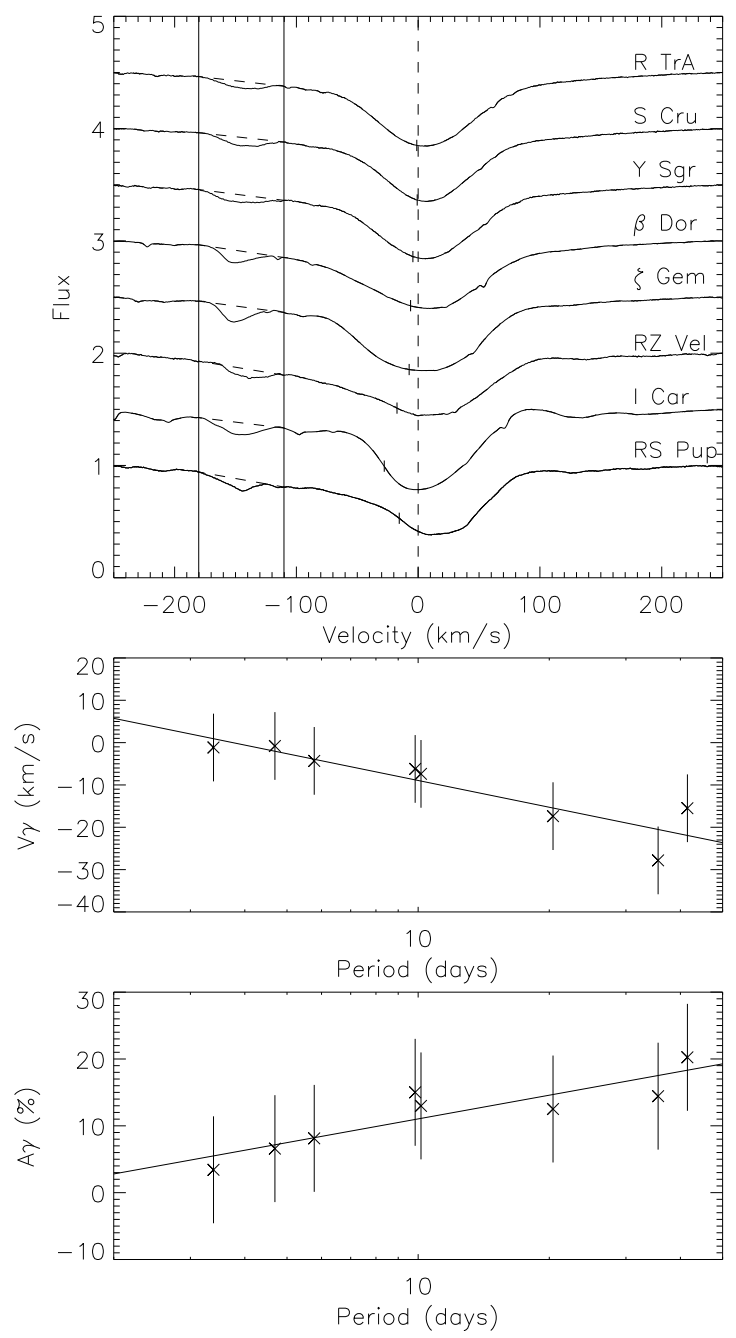

Fig. 5. For each map of Figs. 2-4 an average spectral line profile is calculated. The resulting profiles (arbitrarily shifted by 0.5 in flux) are presented by increasing period on the upper panel of this figure. The metallic line profile between -180 and $-110 \mathrm{~km} \mathrm{~s}^{-1}$ is removed using a linear interpolation (dashed lines), and for each star we derive the first moment of the average spectral line profile $\left(V_{\gamma}\right.$, indicated on each profile by a vertical line) and the spectral line asymmetry $\left(A_{\gamma}\right)$.

Different physical mechanisms can be proposed to explain non-zero $\gamma$-asymmetries (or -velocities): (1) the dynamical structure of the Cepheids' atmosphere and in particular a velocity gradient, and (2) the differential velocity of the line-forming region compared to the corresponding mass elements. However, it has been shown that the $\gamma$-asymmetries averaged over 17 metallic spectral lines (Table 1, Paper II) are only a few percent, and they show a decrease with the period of the Cepheid (Fig. 5, Paper III). In comparison, $\gamma$-asymmetries measured for the $\mathrm{H} \alpha$ line profiles increase with the period and reach $20 \%$ for long-period Cepheids. As a consequence, we suggest that $\gamma$-asymmetries (or -velocities) corresponding to metallic and $\mathrm{H} \alpha$ lines are the result of different physical mechanisms. Then, even if the $\gamma$-velocities of $\mathrm{H} \alpha$ line profiles might be partially due to the dynamical structure of the Cepheids' atmosphere, it seems reasonable to also invoke some possible permanent mass loss from Cepheids with typical velocities projected on the lineof-sight up to $-20 \mathrm{~km} \mathrm{~s}^{-1}$. Moreover, it should be mentioned that our results do not exclude a variation in the mass loss rate with the pulsation phase. Historically, Deasy (1988) examined the 


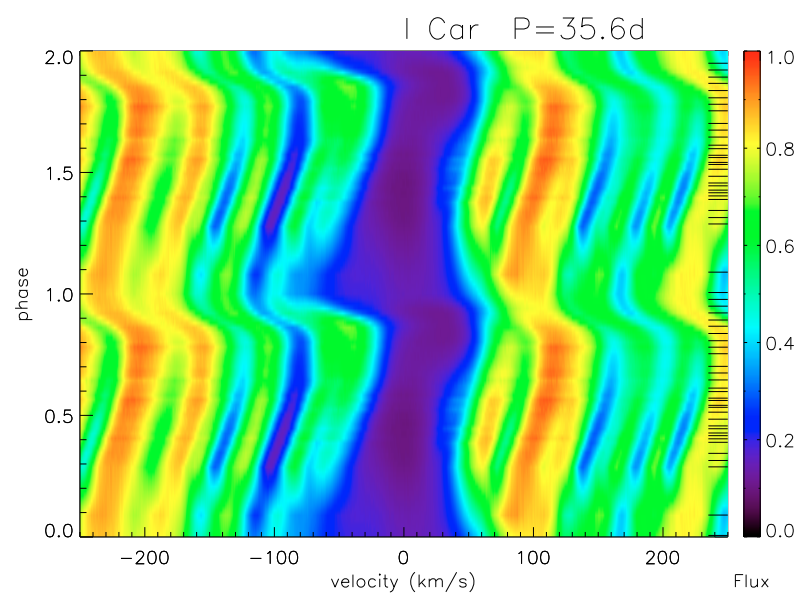

Fig. 6. Time variation of the $\mathrm{H} \beta$ profile of $\ell$ Car in the $[-250$, $250] \mathrm{km} \mathrm{s}^{-1}$ velocity range.

evidence of mass loss from classical Cepheid variables based on infrared and ultraviolet observations. Mass loss rates derived for several stars range between $10^{-10} M_{\odot} \mathrm{yr}^{-1}$ and $10^{-6} M_{\odot} \mathrm{yr}^{-1}$. Moreover, the mass loss seems greater in long-period Cepheids (Willson et al. 1988; Kervella et al. 2008), which is consistent with our results.

Interestingly, from a careful analysis of the period- $V_{\gamma}$ relationship, and taking the errors into account, it is also possible that the stars are separated into two groups, with $V_{\gamma} \simeq 0 \mathrm{~km} \mathrm{~s}^{-1}$ (for $P<10$ days) and $V_{\gamma} \simeq-20 \mathrm{~km} \mathrm{~s}^{-1}$ (for $P>20$ days). If this is true, and the high absolute value of $V_{\gamma}$ for long-period Cepheids is indeed due to permanent mass loss, there might be a minimum threshold value of the period to initiate the permanent mass loss.

The $\mathrm{H} \delta, \mathrm{H} \gamma$, and $\mathrm{H} \beta$ hydrogen spectral lines, which are expected to form between the metallic and the $\mathrm{H} \alpha$ line-forming regions, are unfortunately extremely blended, which prevents us from applying our 1D or 2D-methods for determining $V_{\gamma}$ and $A_{\gamma}$ satisfactorily. As an example, the $\mathrm{H} \beta$ profile of $\ell$ Car is presented in Fig. 6.

To interpret these relationships and to tell which part is related to the dynamical structure of the Cepheids' atmosphere and which to a permanent mass loss, fully consistent hydrodynamical models are required, including pulsating and evolutionary theories, convective energy transport, adaptive numerical meshes, and a refined calculation of the radiative transfer within the pulsating atmosphere and also in the expected circumstellar envelope of the Cepheids. Such detailed modeling is beyond the scope of this paper.

\section{The particular case of $\ell$ Car}

The long period Cepheid $\ell$ Car has the largest apparent angular diameter on the sky, allowing high-precision spectrointerferometric measurements. Thus, $\ell$ Car is an ideal candidate for further observational and theoretical investigations.

Historically, Dawe (1969) showed that the Ca II $k$ line has one blue emission and two absorption components that are not all directly linked to pulsation. In particular, the material seems to be accelerated outwards, which suggests an expansion or an outflow. Schmidt \& Parsons (1984) inferred from the variation of $\mathrm{Mg}$ II $h$ and $k$ profiles that the central absorption has a circumstellar origin, while the increase in the emission around phase of light maximum is related to a shock wave. This latter is confirmed by Böhm-Vitense \& Love (1994), who estimated a shock velocity of $100 \mathrm{~km} \mathrm{~s}^{-1}$, i.e., on the order of the escape velocity: mass loss is enabled from pulsation, as also pointed out by Deasy (1988). A closer look at the Mg II profiles led the authors to suggest that the quantity of mass ejected at each pulsation period is different. However, Taylor et al. (1997) see no cycleto-cycle variations in the low photosphere where metallic lines are formed. Hence, interactions between the propagating wave and the atmosphere seem to play a major role. Böhm-Vitense $\&$ Love (1994) also pointed out the presence of a circumstellar shell and emission blobs in O I profiles at a distance of about 1000 AU from the star. Baldry et al. (1997) confirmed the presence of mass loss and of a circumstellar shell from the variation in the $\mathrm{H} \alpha$ line.

From our observation (Fig. 4), we find that the $\mathrm{H} \alpha$ profile of $\ell$ Car is composed of:

- an absorption component more or less following the pulsation of the star;

- a dominant absorption component whose velocity (pixel corresponding to the minimum of the spectral line) is nearly zero $\mathrm{km} \mathrm{s}^{-1}$ in the stellar rest frame (Rodgers \& Bell 1968; Baldry et al. 1997). This means it does not take part in the pulsation, and we attribute this component to a circumstellar shell, which is certainly the result of the on going permanent mass loss of $\ell$ Car. This component might also be present for other Cepheids, even if it should then be quite weak since it is not observed in the data;

- near $\phi=0$, a red emission component appears (around $100 \mathrm{~km} \mathrm{~s}^{-1}$ ) whose velocity and intensity slowly decreases until $\phi=0.5$. This red emission has a maximum of $1.17 \pm$ 0.01 ( $17 \%$ of the continuum) near $\phi=0.08$;

- near $\phi=0.6$, a blue emission appears (around $-55 \mathrm{~km} \mathrm{~s}^{-1}$ ) and reaches its peak at $\phi=0.84$ with a maximum in flux of $1.1 \pm 0.01$, and decreases again up to phase 0.9 ;

- the positive asymmetry of the $\mathrm{H} \alpha$ line (especially at phases between 0.0 and 0.3 ) is certainly related to some permanent mass loss around the star as discussed in Sect. 3.

In contrast to $\ell$ Car, RZ Vel, and RS Pup show very weak red emission components, respectively $1.02 \pm 0.01$ and $1.01 \pm 0.01$. Such $\mathrm{H} \alpha$ spectral line characterization is extremely important to support future hydrodynamical modeling.

\section{Conclusion}

We presented very high-quality time series of $\mathrm{H} \alpha$ profile variations of Cepheids. For the first time, is was possible to derive 2-dimensional wavelength versus time maps. The short-period Cepheids show typical $\mathrm{H} \alpha$ line profiles that follow the pulsating envelope of the star, while long-period Cepheids exhibit a more complex spectroscopic behavior. Using these maps, we found new relationships between the period of Cepheids and their $\gamma$-velocities and asymmetries. These results may be related to the whole dynamical structure of Cepheids and also to some permanent mass loss in Cepheids. This kind of relationship, together with the shape of $\mathrm{H} \alpha$ profiles, remain extremely difficult to explain and require further theoretical investigations, which include the hydrodynamics and radiative transfer in the pulsating atmospheres of Cepheids and their circumstellar envelopes.

Acknowledgements. Based on observations collected at La Silla Observatory, Chile, in the framework of European Southern Observatory's programs 072.D0419 and 073.D-0136. We thanks D. Bersier, P. Kervella, and P. Mathias 
for useful discussions, and in particular P. Kervella for having provided the HARPS data. N.N., J.H.G., and F.M. acknowledge the Max Planck Institut for Radioastronomy for financial support.

\section{References}

Baldry, I. K., Taylor, M. M., Bedding, T. R., \& Booth, A. J. 1994, MNRAS, 289, 979

Böhm-Vitense, E., \& Love, S. G. 1994, ApJ, 420, 401

Burki, G., Mayor, M., \& Benz, W. 1982, A\&A, 109, 258

Deasy, H. P. 1988, MNRAS, 231, 673

Dawe, J. A. 1969, MNRAS, 145, 377

Fadeyev, Y. A., \& Gillet, D. 2004, A\&A, 420, 423

Fernie, J. D., Beattie, B., Evans, N. R., \& Seager, S. 1995, IBVS, 4148

Freedman, W., Madore, B. F., Gibson, B. K., et al. 2001, ApJ, 553, 47

Fouqué, P., Arriagada, P., Storm, J., et al. 2007, A\&A, 476, 73

Kervella, P., Mérand, A., Perrin, G., \& Coudé Du Foresto, V. 2006, A\&A, 448, 623

Kervella, P., Mérand, A., Szabado, L., et al. 2008, A\&A, 480, 167

Kovacs, G., Kisvarsanyi, E. G., \& Buchler, J. R. 1990, ApJ, 351, 606

Grenfell, T. C., \& Wallerstein, G. 1969, PASP, 81, 732
McAlary, C. W., \& Welch, D. L. 1986, AJ, 91, 1209

Mathias, P., Gillet, D., Fokin, A., et al. 2006, A\&A, 457, 575

Mérand, A., Kervella, P., Coudé du Foresto, V., et al. 2006, A\&A, 453, 155

Mérand, A., Aufdenberg, J. P., Kervella, P., et al. 2007, ApJ, 664, 1093

Nardetto, N., Fokin, A., Mourard, D., et al. 2004, A\&A, 428, 131

Nardetto, N., Mourard, D., Kervella, P., et al. 2006, A\&A, 453, 309 (Paper I)

Nardetto, N., Mourard, D., Mathias, Ph., et al. 2007a, A\&A, 471, 661 (Paper II)

Nardetto, N., Mourard, D., Kervella, P., et al. 2007b, yCat, 34530309

Nardetto, N., Stoekl, A., Bersier, D., \& Barnes, T. G. 2008, 489, 1255 (Paper III)

Renka, R. 1982, Interpolation of Data on the Surface of a Sphere, Oak Ridge National Laboratory Report ORNL/CSD-108

Rodgers, A. W., \& Bell, R. A. 1968, MNRAS, 138, 23

Sabbey, C. N., Sasselov, D. D., Fieldus, M. S., et al. 1995, ApJ, 446, 250

Sasselov, D. D., \& Karovska, M. 1994, ApJ, 432, 367

Schmidt, E. G., \& Parsons, S. B. 1984, ApJ, 279, 202

Taylor, M. M., Albrow, M., Booth, A. J., et al. 1997, MNRAS, 292, 662

Wallerstein, G. 1972, PASP, 84, 656

Wallerstein, G. 1979, PASP, 91, 772

Wallerstein, G. 1983, PASP, 95, 422

Wallerstein, G., Jacobsen, T. S., Cottrell, P. L., Clark, M., \& Albrow, M. 1992, MNRAS, 259, 474

Willson, L. A., Bowen, G. H., \& Struck-Marcell, C. 1988, ComAp, 12, 17 